\title{
ACTUATOR FAULT DIAGNOSIS FOR FLAT SYSTEMS: A CONSTRAINT SATISFACTION APPROACH
}

\author{
RAMATOU SEYDOU * , TAREK RAISSI ${ }^{* *}$, Ali ZOLGHADRI* , DENIS EFIMOV*** \\ * Automatic Control Group, IMS-Lab \\ Bordeaux University, 351 cours de la libération, 33405 Talence cedex, France \\ e-mail \{ramatou.seydou, ali.zolghadri\}@ims-bordeaux.fr \\ ${ }^{* *}$ CEDRIC-Lab \\ National Conservatory of Arts and Crafts, 292, rue Saint-Martin, 75141 Paris, France \\ e-mail: tarek.raissi@cnam.fr \\ *** Non-A Project INRIA-LNE \\ Parc scientifique de la haute borne 40, Av. Halley, Bât. A, Park Plaza, 59650 Villeneuve d'Ascq, France \\ e-mail: denis.efimov@inria.fr
}

\begin{abstract}
This paper describes a robust set-membership-based Fault Detection and Isolation (FDI) technique for a particular class of nonlinear systems, the so-called flat systems. The proposed strategy consists in checking if the expected input value belongs to an estimated feasible set computed using the system model and the derivatives of the measured output vector. The output derivatives are computed using a numerical differentiator. The set-membership estimator design for the input vector takes into account the measurement noise thereby making the consistency test robust. The performances of the proposed strategy are illustrated through a three-tank system simulation affected by actuator faults.
\end{abstract}

Keywords: fault detection, input observer, flat systems, consistency techniques.

\section{Introduction and problem setting}

The theory related to model-based FDI has been developed since the 1970s and can be considered today a mature and well-structured field of research within the control community (for a survey, see Chen and Patton, 1999; Ding, 2008; Korbicz et al., 2004). The role of the FDI unit is to detect, isolate and estimate the severity of a fault. The IFAC SAFEPROCESS Technical Committee defines a fault as an unpermitted deviation of at least one characteristic property or parameter of the system from the standard conditions (Isermann and Ball, 1997). Such malfunctions may occur in sensors, actuators or other devices and affect adversely the local or global behavior of the system.

Generally, the main desirable characteristics of an FDI system are

- early detection and identification of abnormal situations, i.e., detection delay should be minimized;

- good ability to discriminate between different failures (isolability);

- good robustness to various noise and sources of uncertainty, and their propagation through the system;

- high sensitivity and performance, i.e., high detection rate and low false alarm rate.

The basic structure of a classical model-based FDI technique is depicted in Fig. 1

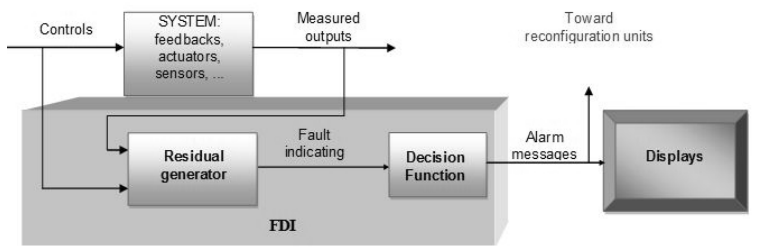

Fig. 1. Basic structure of FDI.

The core element is residual generation and many 
approaches concentrate on this step (Fig. 1). Note that if only fault detection is of interest, reconstructing the fault rather than detecting its presence through a residual signal can be a nice alternative solution. Residual evaluation and decision making consist in checking the residuals and triggering alarm messages if the tolerances are exceeded. The thresholds can be set into different kinds.

The simplest way is to use a constant threshold. The main advantage with fixed thresholds is their simplicity and reliability. To enhance the robustness of FDI schemes against small parameter variations and disturbances during residual generation, various design and evaluation tools have been proposed (Hwang et al., 2010). Robust FDI can be achieved if the residual signals maintain these sensitivity properties over a suitable range of system dynamics.

Most of the well known approaches assume the availability of an LTI model or specific nonlinearities. For systems with strong nonlinear behavior, several FDI methodologies exist in the literature, including LPV transformations (Bokor and Balas, 2004; Henry and Zolghadri, 2004; Grenaille et al., 2008), adaptive observers (Ding and Frank, 1993; Wang and Daley, 1996; Wang et al., 1997; Chen et al., 2000; Besançon and Zhang, 2002; Caccavale and Villani, 2004; Liu, 2009), stochastic techniques (Wang and Noriega, 2001; Wang and Lin, 2000; Wang, 2003; Poulsen and Niemann, 2008), algebraic approaches (Zhang et al., 1998; Berdjag et al., 2006) or sliding mode-based methods (Edwards and Spurgeon, 2000; Hakiki et al., 2006; Yan and Edwards, 2008). For a good survey, see the work of Bokor and Szab (2009) and the references therein.

Most of the proposed approaches make use of particular structural characteristics of a given nonlinear system to generate output residuals. Typically, the observer design problem is solvable if the system model can be transformed into a canonical form, which may be an assumption difficult to satisfy in many applications. Another approach is based on input observer design. In this approach, the input observer is a dynamical system with an output that converges asymptotically or in finite time to a fault to be detected (Corless and Tu, 1998; Ha and Trinh, 2004). More recently, set-membership fault detection techniques have been investigated by several authors (Puig et al., 2003; Stancu et al., 2005; Fagarasana et al., 2004; Ragot et al., 2006; Lalami and Combastel, 2006; Raïssi et al., 2010). Many publications are based on interval observers (Gouzé et al., 2000; Bernard and Gouzé, 2004; Moisan and Bernard, 2006; Moisan et al., 2009), where the idea was to design two-point observers to compute an upper and a lower bound for the state vector domain under both stability and cooperativity conditions. An interval residual is given as the difference between the predicted set from the model and the real value measured by the sensors. Another approach reported by
Ingimundarson et al. (2009) and Puig (2010) is based on state/parametric consistency checks.

This paper investigates a set-membership fault detection technique for a particular class of nonlinear systems, the so-called flat ones (Fliess et al., 1992). The proposed approach belongs to the class of input fault detection techniques. It consists in formulating the input estimation issue using a Constraint Satisfaction Problem (CSP) (Neumaier, 2004; Arangú and Salido, 2011). A CSP input estimator is then designed using the relation between the input vector and the flat outputs. This observer requires the computation of a finite number of flat output derivatives. The robustness is achieved by taking into account the whole domain of the uncertainties (measurement noise) and without any linearization. This technique is well suited to deal with actuator faults as well as parametric or sensor faults. However, in this paper, we choose to focus on actuator faults. The methodology includes a fault identification stage where interval residual quantities are generated by calculating the gap between the estimated input set and the measured input value at each time instant.

The paper is structured as follows. Section 2 recalls some basic notations which will be used throughout the paper. The problem is formulated in Sections 3 and 4. Then, the CSP input estimator is presented and used in the FDI procedure (Section 5). Finally, the methodology is illustrated through a three-tank system application, and some concluding remarks are given.

\section{Preliminaries}

This section recalls some basic and useful definitions.

2.1. Flatness. Consider a nonlinear system described by

$$
\left\{\begin{array}{l}
\dot{x}=f(x)+g(x) u, \\
y=h(x)
\end{array}\right.
$$

where $x \in \mathbb{R}^{n}, y \in \mathbb{R}^{m}, u \in \mathbb{R}^{m}$, and assume $f: \mathbb{R}^{n} \rightarrow$ $\mathbb{R}^{n} g: \mathbb{R}^{n} \rightarrow \mathbb{R}^{n \times m}$ are two smooth vector fields while $h: \mathbb{R}^{n} \rightarrow \mathbb{R}^{m}$ is a differentiable smooth function. The system (1) is said to be flat with a flat output vector $y$ if and only if one can describe the system states and inputs $x, u$ only from the flat outputs and a finite number of their derivatives, i.e.

$$
\left\{\begin{array}{l}
x=\theta\left(y, \dot{y}, \ldots, y^{p}\right), \\
u=s\left(y, \dot{y}, \ldots, y^{p+1}\right),
\end{array}\right.
$$

where $\theta$ and $s$ are two smooth vector fields. 
2.2. Nonlinear local observability. The matrix

$$
H(x)=\left(\begin{array}{c}
\mathrm{d} h(x) \\
\mathrm{d} L_{f} h(x) \\
\vdots \\
\mathrm{d} L_{f}^{n-1} h(x)
\end{array}\right)
$$

is called the local observability matrix for 11 where $L_{f} h(x)$ is the Lie derivative of the function $h$ along the vector field $f$ and $d L_{f} h(x)$ is the gradient $\frac{\partial L_{f} h(x)}{\partial x}$ (Hermann and Krener, 1977). The system (1) satisfies the observability rank condition at the point $x$ if the observability matrix has full rank, i.e. $\operatorname{rank}(H(x)=n$.

2.3. Interval tools. A real interval $[a]=[\underline{a}, \bar{a}]$ is a connected and closed subset of $\mathbb{R}$. The set of all real intervals of $\mathbb{R}$ is denoted by $\mathbb{I} \mathbb{R}$. Real arithmetic operations are extended to intervals (Moore, 1966; Hansen, 2004). Let $f: \mathbb{R}^{n} \rightarrow \mathbb{R}^{m}$. The range of the function $f$ over an interval vector $[x]$ is given by $f([x])=\{f(x) \mid x \in[x]\}$. An interval function $[f]: \mathbb{R}^{n} \rightarrow \mathbb{R}^{m}$ is an inclusion function for $f$ if $\forall[x] \in \mathbb{R}^{n}, f([x]) \subseteq[f]([x])$.

2.4. Constraint satisfaction problems. A constraint satisfaction problem (CSP) is defined by a set of variables, $X_{1}, X_{2}, \ldots, X_{n}$, and a set of constraints, $C_{1}, C_{2}, \ldots, C_{m}$. Each variable $X_{i}$ has a nonempty domain $D_{i}$ of possible values. Each constraint $C_{i}$ involves some subset of the variables and specifies the allowable combinations of values for that subset. A state of the problem is defined by an assignment of values to some or all of the variables, $\left\{X_{i}=v_{i}, X_{j}=v_{j}, \ldots\right\}$ (Neumaier, 2004).

Constraint propagation is a way to solve CSPs. The aim of propagation techniques is to contract as much as possible the domains for the variables without losing any solution (Waltz, 1975). When interval uncertainties are considered, consistency methods combining interval and constraint satisfaction techniques can be used to deal with problems such as parameter/state estimation and further fault detection problems.

In the case of flat systems, CSP variables correspond to the states and inputs related to the flat output by a specific and unique map. This map defines the constraints. In order to retrieve the state and input vectors satisfying the constraints, consistency techniques are applied through flatness equations.

\section{Problem statement}

Consider a system described by (1) and assume that the measurement error $e$ is bounded with a known bound $\bar{e}$.
The output vector $y$ belongs to

$$
y \in\left[y_{m}-\bar{e}, y_{m}+\bar{e}\right]
$$

where $y_{m}$ is the measured signal.

Assumption 1. The actuator fault $f_{a}$ is modeled as follows:

$$
u=u_{0}+f_{a}
$$

where $u_{0}$ is the nominal input.

This assumption corresponds to additive fault modeling. Note that multiplicative faults can be modeled as additive faults considering the transformation described by Ding (2008). Actually, additive faults do not affect system stability under feedback control whereas multiplicative ones could. The additive fault assumption is made throughout the paper since it is easy to derive the fault $f_{a}$ from Eqn. (5). Nevertheless, this assumption can be relaxed without any additional theoretical developments since the interval technique used to solve the CSP (13) does not need such assumptions.

It is required to estimate the fault using the output vector and its derivatives. To solve the problem, it is proposed to rewrite the second equation of (2) expressing the input $u$ as a polynomial function of the flat output $y$ and a finite number of its derivatives. This step is based on the parity space approach for polynomial nonlinear systems. The resulting relations between the variables (input, flat outputs and their derivatives, fault) constitute the constraints for the CSP input estimator used in the FDI procedure (Sections 5 and 6). The numerical output differentiation for $y$ is performed using a Higher Order Sliding Modes (HOSM) differentiator. Both fault detection and fault identification phases are based on input estimation through a CSP input estimator.

The common point between the presented approach and the geometric methodology proposed by De Persis and Isidori $(2001 ; 2002)$ is a kind of model invalidation. In this paper, the model is invalidated through the use of a consistency test whereas the geometric approach consists mainly in finding an unobservable subspace due to the occurrence of a certain fault among others. Note that this geometric approach for fault detection and isolation relies on stochastic assumptions.

\section{Parity space and flat systems}

The aim of this section is to derive relations between $u_{0}$, $y$ and $f_{a}$ for the system (1). Taking the derivatives of the 
output vector up to an order $n-1$, we obtain

$$
\left\{\begin{aligned}
y & =y^{(0)}=h(x), \\
y^{(1)} & =\frac{\partial h(x)}{\partial t}=\frac{\partial h(x)}{\partial x} \cdot \frac{\partial x}{\partial t} \\
& =\frac{\partial h(x)}{\partial x} \cdot[f(x)+g(x) u] \\
& =L_{f} h(x)+L_{g} h(x) \cdot u, \\
y^{(2)} & =L_{f}^{2} h(x)+L_{g} L_{f} h(x) \cdot u+L_{f} L_{g} h(x) \cdot u \\
& +L_{g}^{2} h(x) \cdot u u^{T}+L_{g} h(x) \cdot u^{(1)}, \\
& \vdots
\end{aligned}\right.
$$

Equation (4) can be rewritten as

$$
Y=H+G W
$$

where

$$
\begin{gathered}
Y=\left(\begin{array}{c}
y \\
y^{(1)} \\
y^{(2)} \\
\vdots
\end{array}\right), \quad H=\left(\begin{array}{c}
L_{f} h(x) \\
L_{f}^{1} h(x) \\
L_{f}^{2} h(x) \\
\vdots
\end{array}\right), \\
W=\left(\begin{array}{c}
u \\
u \cdot u^{T} \\
u^{(1)} \\
\vdots
\end{array}\right)
\end{gathered}
$$

and

$$
G=\left(\begin{array}{ccc}
0 & 0 & 0 \\
L_{g} h(x) & 0 & 0 \\
L_{g} L_{f} h(x)+L_{f} L_{g} h(x) & L_{g}^{2} h(x) & L_{g} h(x) \\
\vdots & \vdots & \vdots \\
& 0 & \cdots \\
\cdots & \cdots \\
0 & \cdots \\
\vdots & \vdots
\end{array}\right) .
$$

Equation (6) shows that the consecutive derivatives of the measured output vector are polynomial functions of the derivatives of the input vector $u$ (Sontag and Wang, 1991). The relation (6) can be formulated as

$$
\begin{aligned}
& \phi_{i}\left(x, u, \ldots, u^{(i-2)}, y^{(i-1)}\right) \\
& \quad=y^{(i-1)}-L_{F}^{i-1} h(x)=0, \quad i=1, \ldots, n,
\end{aligned}
$$

where $\phi_{i}$ is a nonlinear function of its arguments and $F(x, u)=f(x)+g(x) u$.

Under the observability rank condition (3) and using the implicit functions theorem (Krantz and Parks, 2002), Eqn. (7) has a solution,

$$
x=P\left(u^{(0)}, y^{(0)} \ldots, u^{(n-1)}, y^{(n-1)}\right) .
$$

Thus, the state $x$ is a function of the input and output vector derivatives, transforming (7) into

$$
\begin{aligned}
\Psi_{i}\left(u, y, u^{(1)}, \ldots, y^{(1)}, \ldots\right) & \\
=\phi_{i}( & P\left(u, y \ldots, u^{(n-1)}, y^{(n-1)}\right), u \\
\left.\ldots, u^{(n-2)}, y^{(n-1)}\right)=0 & \\
& i=1,2, \ldots,
\end{aligned}
$$

where $\Psi_{i}$ is a nonlinear function of its arguments. Taking into account the expression of $u$ given by (5), the relation (9) can be transformed into

$$
\Psi_{i}\left(\left(u_{0}+f_{a}\right), y, \ldots,\left(u_{0}+f_{a}\right)^{\left(\alpha_{i}\right)}, y^{\left(\alpha_{i}\right)}\right)=0 .
$$

The relation (10) is established for a general class of nonlinear systems. Nevertheless, it is well known that for flat systems the input is related to the output and its derivatives as

$$
u=s\left(y, y^{(1)}, \ldots, y^{(p+1)}\right) .
$$

Thus, the relations (9) and (11) lead to

$$
\begin{aligned}
\Psi_{l}\left(\left(u_{0}+f_{a}\right), y, y^{(1)}, \ldots, y^{(p+1)}\right) & =0, \\
l & =1, \ldots, m .
\end{aligned}
$$

Remark 1. For simplicity, only additive faults are considered in this paper. Nevertheless, multiplicative faults modeled by $u=u_{0}(1+\alpha)$, where $\alpha$ is the fault effect, can also be studied without any additional investigations.

\section{Input estimation and fault diagnosis}

Given a flat system described by (1) and admitting the decoupling relation (12), the input estimation problem is formulated as a CSP given by

$$
\left\{\begin{array}{l}
\Psi_{l}\left(u, y, y^{(1)}, \ldots, y^{(p+1)}\right)=0, l=1, \ldots, m, \\
y \in[y], y^{(1)} \in\left[y^{(1)}\right], \ldots, y^{(p+1)} \in\left[y^{(p+1)}\right] \\
u \in U,
\end{array}\right.
$$

which can be solved using interval analysis. Note that a similar formulation has been performed for state estimation by Jaulin (2013), according to whom the output derivatives are supposed to be measured. This is not the case in our work. Finding solutions to (13) needs an evaluation of the measurement derivatives. In the following, the measurement derivatives are estimated using HOSM differentiators (Levant, 1998; 2003).

5.1. HOSM differentiator for derivative estimation. Let $y_{i}$ be the signal to be differentiated and $z_{0}, z_{1}, \ldots, z_{n}$ be some estimates for the signal $y_{i}$ and its derivatives. 
Note that $y_{i}$ is the $i$-th output vector component and $y_{i}=y_{i_{0}}+e_{i} ; e_{i}$ is a bounded Lebesgue-measurable noise with unknown features and an unknown base signal $y_{i_{0}}$ with the $n$-th derivative having a known Lipschitz constant $C_{i}>0$. The $n$-th-order HOSM differentiator is given by

$$
\left\{\begin{array}{l}
\dot{z}_{0}=\nu_{0}, \\
\nu_{0}=-\alpha_{0}\left|z_{0}-y_{i}\right|^{\frac{n}{n+1}} \operatorname{sign}\left(z_{0}-y_{i}\right)+z_{1}, \\
\dot{z}_{1}=\nu_{1}, \\
\nu_{1}=-\alpha_{1}\left|z_{1}-\nu_{0}\right|^{\frac{n-1}{n}} \operatorname{sign}\left(z_{1}-\nu_{0}\right)+z_{2}, \\
\vdots \\
\dot{z}_{k}=\nu_{k}, \\
\nu_{k}=-\alpha_{k}\left|z_{k}-\nu_{k-1}\right|^{\frac{n-k}{n-k+1}} \operatorname{sign}\left(z_{k}-\nu_{k-1}\right) \\
+z_{k+1} \\
\vdots \\
\dot{z}_{n}=-\alpha_{n} \operatorname{sign}\left(z_{n}-\nu_{n-1}\right) .
\end{array}\right.
$$

It has been proved (Levant, 2001) that the best estimate accuracy of the $k$-th derivative is proportional to

$$
a c c_{i_{k}}=\mu_{i_{k}} C_{i}^{\frac{k}{n+1}} \bar{e}_{i}^{\frac{n+1-k}{n+1}}, \quad k=0, \ldots, n,
$$

when the Lipschitz constant of the $n$-th derivative of the clear-off-noise signal is bounded by a certain constant $C_{i}$ and $\mu_{i_{k}} \geq 1\left(\mu_{k}\right.$ depends only on $\alpha_{k}, k=0, \ldots, n$. Please refer to Proposition 1 of Levant (2001) for more details). Hence, the derivative domain is $y_{i}^{(k)} \in\left[y_{i_{e} s t}^{(k)}-\right.$ $\left.a c c_{i_{k}}, y_{i_{e} s t}^{(k)}+a c c_{i_{k}}\right]$ where $y_{i_{e} s t}^{(k)}=z_{k}$ is the estimate of the $k$-th derivative for $y_{i}$.

5.1.1. Input estimation. At each time instant $t_{j}$, denote by $U_{j}$ the domain of $u$ at $t_{j}$. If no prior information about the domain of $u$ is available, we can select the domain $\left.U_{j}=\right]-\infty,+\infty[$. Thus, the basics of the input estimation method consist in computing all the values of $u$ satisfying (13). The idea is to remove parts of the searching domain $U_{j}$ for the model input that are inconsistent with the measured data $y_{j}$ and their derivatives up to an order $p+1$. For easy reference, the main steps of the input estimation are summarized as Algorithm 1.

Note that $U$ is the initial search domain for the input vector and SIVIAP is a set inversion algorithm which makes is possible to solve CSPs in a guaranteed way using interval analysis (Jaulin et al., 2001).

Remark 2. The vectors $\bar{e}, a c c_{k}$ and $y^{(k)}$ should be respectively understood as $\bar{e}=\left(\bar{e}_{1}, \ldots, \bar{e}_{m}\right)^{T}, a c c_{k}=$ $\left(a c c_{1_{k}}, \ldots, a c c_{m_{k}}\right)^{T}$, and $y^{(k)}=\left(y_{1}^{(k)}, \ldots, y_{m}^{(k)}\right)^{T}$, where $m$ represents the flat output vector dimension.

5.1.2. Residual generation and fault diagnosis. The residual generation and fault detection procedures are
Algorithm 1. CSP Estimator.

Step 0. (Inputs: $\left.y\left(t_{j}\right), j=1, \ldots, N, U\right)$, Output: $\left[u\left(t_{j}\right)\right]$ )

Step 1. Flatness modelling as a CSP (13).

Step 2. For $j=1, \ldots, N$, do

Step 2.1. Estimate the derivatives $y^{(k)}, k=1, \ldots, p+1$ using (14).

Step 2.2. Estimate the bound $a c c_{k}$ and construct the domains of $y\left(t_{j}\right)$ and its derivatives.

Step 2.3 Solve the CSP using the SIVIAP algorithm to obtain $\left[u\left(t_{j}\right)\right]$.

based on input estimation. At each time instant $t_{j}$, we consider the algorithm output $\left[u\left(t_{j}\right)\right]$ and an inclusion test is run over an interval residual. This residual denotes the gap between the estimated input set and the expected input value. The lower (respectively, upper) bound of the residual corresponds to the difference between the estimated set lower (respectively, upper) bound input $u$ and the value of the fault free model input $u_{0}$. The residual is defined by

$$
\left[r\left(t_{j}\right)\right]=\left[\underline{u}\left(t_{j}\right)-u_{0}\left(t_{j}\right), \bar{u}\left(t_{j}\right)-u_{0}\left(t_{j}\right)\right] .
$$

The consistency test aim is to check whether the expected (fault-free case or controller output) input value $u_{0}\left(t_{j}\right)$ belongs to the estimated domain $\left[u\left(t_{j}\right)\right]$, i.e.,

$$
\operatorname{det}_{j}=\left\{\begin{array}{cc}
0, & 0 \in\left[r\left(t_{j}\right)\right], \\
1, & 0 \notin\left[r\left(t_{j}\right)\right],
\end{array}\right.
$$

which is equivalent to checking

$$
\operatorname{det}=\left\{\begin{array}{cc}
0, & u_{0}\left(t_{j}\right) \in\left[u\left(t_{j}\right)\right], \\
1, & u_{0}\left(t_{j}\right) \notin\left[u\left(t_{j}\right)\right] .
\end{array}\right.
$$

The algorithm used for input estimation could involve several bisections over the initial input domain for the admissible value research. Actually, the computing time increases exponentially with the input dimension (Jaulin et al., 2001). In the fault detection phase, we only check whether the actual input $u_{0}$ is consistent with the measurements. If a fault is detected, the algorithm CSP Estimator will be applied to estimate its amplitude.

5.1.3. Fault identification. Fault identification is the characterization of the fault besides its occurrence time. This task is here restricted to the determination of the fault amplitude. Since we consider additive actuator faults, the fault amplitude or, more precisely, the estimated fault domain is given by the residual expression

$$
\left[\underline{f}_{a}, \bar{f}_{a}\right]=[r]=\left[\underline{u}-u_{0}, \bar{u}-u_{0}\right] .
$$

Note that the approaches developed by Jiang et al. (2004), Akhenak et al. (2003), Kabore and Wang (2001) 
as well as Orani et al. (2009) propose alternative solutions for actuator failures and simultaneous faults diagnosis. These techniques are based on a robust sliding mode observer design whereas the sliding mode concept is used in this paper for robust numerical differentiation of bounded noisy measurements. Most of these methodologies require system linearizations (Akhenak et al., 2003; Kabore and Wang, 2001) or a canonical form (Jiang et al., 2004). The technique proposed in this paper considers the original nonlinear model without any linearization and the results are guaranteed by the fact that the variables are modeled by means of intervals containing the actual value which cannot be known exactly. This is one of the main advantages of set-membership techniques. However, the computational burden remains a drawback when dealing with high dimensional systems.

5.1.4. Minimum detectable fault. The minimum detectable fault corresponds to the smallest amplitude for a fault to be detectable with respect to the measurement error bounds $\bar{e}_{i}$ and output derivatives error bounds $a c c_{i_{k}}$. Recall that the bound $a c c_{k}$ is given by

$$
a c c_{k}=\mu_{k} \cdot C^{\frac{k}{n+1}} \cdot \bar{e}^{\left(\frac{n+1-k}{n+1}\right)}, \quad k=0, \ldots, n .
$$

When no fault occurs, the residual $[r]$ is centered around the value 0 and its upper and lower bounds ( $\bar{r}$ and $\underline{r}$ ) can be computed from the residual expression and the bounds $a c c_{k}$ :

$$
\left\{\begin{array}{l}
{[r]=[\underline{r}, \bar{r}]=\left[\underline{u}-u_{0}, \bar{u}-u_{0}\right],} \\
{[\underline{r}, \bar{r}]=[s]\left([y],\left[y^{(1)}\right], \ldots,\left[y^{(p+1)}\right]\right)-u_{0} .}
\end{array}\right.
$$

In a fault-free case, we have $\bar{r} \geq 0$ (respectively $\underline{r} \leq 0)$. Thus, the minimum detectable fault is the fault that makes $\bar{r}$ (respectively, $\underline{r}$ ) cross the null value towards negative values (respectively, positive values). The minimum detectable fault depends on the nominal input and output amplitudes.

In the following, we assume that the system is subject to null valued inputs and the flat outputs are subsequently null ( $y_{m}=0$ under null initial conditions or in a steady state case). Moreover, we assume that a fault $f_{a}$ occurs at the same time on the actuators. The output domain is then

$y \in\left[y_{m}-\bar{e}, y_{m}+\bar{e}\right] \Rightarrow y \in[-\bar{e},+\bar{e}]=\left[-a c c_{0}, a c c_{0}\right]$.

The residual domain becomes

$[\underline{r}, \bar{r}]=[s]\left(\left[-a c c_{0}, a c c_{0}\right], \ldots,\left[-a c c_{p+1}, a c c_{p+1}\right]\right)-u_{0}$.

Since $u_{0}$ is the nominal input value and is assumed to be null, the residual domain is

$$
[\underline{r}, \bar{r}]=[s]\left(\left[-a c c_{0}, a c c_{0}\right], \ldots,\left[-a c c_{p+1}, a c c_{p+1}\right]\right) .
$$

Actually, this domain gives roughly the minimum detectable fault $f_{a_{m} i n}$ amplitude:

$$
f_{a_{m} i n}=\min (|\underline{r}|,|\bar{r}|) .
$$

For the general case, the nominal inputs and outputs should be taken into account.

\section{Application: A three tank hydraulic system}

The proposed methodology is illustrated on a well-known hydraulic laboratory system (Fig. 2) which is modeled by

$$
\begin{aligned}
\dot{x}_{1} & =-\frac{a_{13}}{S} \sqrt{x_{1}-x_{3}}+\frac{1}{S} u_{1}, \\
\dot{x}_{2} & =\frac{a_{32}}{S} \sqrt{x_{3}-x_{2}}-\frac{a_{20}}{S} \sqrt{x_{2}}+\frac{1}{S} u_{2}, \\
\dot{x}_{3} & =\frac{a_{13}}{S} \sqrt{x_{1}-x_{3}}-\frac{a_{32}}{S} \sqrt{x_{3}-x_{2}}, \\
y_{1} & =x_{1} \\
y_{2} & =x_{3}
\end{aligned}
$$

where $y_{1}$ and $y_{2}$ are the flat outputs of the system and $x=\left(x_{1}, x_{2}, x_{3}\right)^{T}=\left(h_{1}, h_{2}, h_{3}\right)^{T}$ represents the state vector (under the condition $h_{1}>h_{3}>h_{2}$ for all times), $u=\left(u_{1}, u_{2}\right)^{T}=\left(Q_{1}, Q_{2}\right)^{T}$ is the control vector.

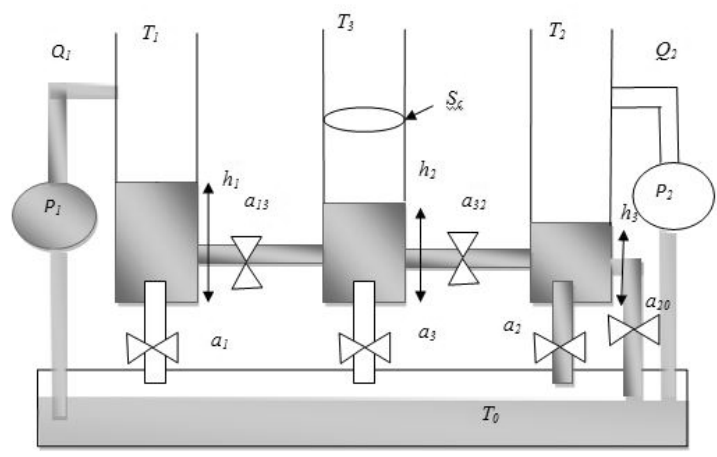

Fig. 2. Three-tank system.

The three-tank system is a popular benchmark used in many published works. Moreover, this example is fully nonlinear and flat, which is important for our approach. This example has been addressed in other papers (Join et al., 2005; Theilliol et al., 2002; Zolghadri et al., 1996), where the authors adopted the assumption that the whole state vector of the plant is available for measurement. The technique proposed in this paper only requires two measurable flat outputs.

The noise magnitude corrupting the measurements $y_{m}$ is bounded by $[ \pm 2] \mathrm{mm}$. The system is under feedback control and two PI controllers have been implemented for 
the flat output $x_{1}$ and the non-flat output $x_{2}$. It is easy to prove the system flatness (and subsequently its local observability) through the following equations:

$$
\left\{\begin{array}{l}
x_{1}=y_{1}, \\
x_{2}=y_{2}-\left(\frac{a_{13} \sqrt{y_{1}-y_{2}}-S_{c} \dot{y}_{2}}{a_{32}}\right)^{2}, \\
x_{3}=y_{2},
\end{array}\right.
$$

and

$$
\left\{\begin{aligned}
u_{1}= & S_{c} \dot{y}_{1}+a_{13} \sqrt{y_{1}-y_{2}}, \\
u_{2}= & -\frac{\frac{2 S_{c}}{a_{32}}\left(a_{13} \sqrt{y_{1}-y_{2}}-S_{c} \dot{y}_{2}\right)}{a_{32}} \\
& \times\left(\frac{a_{13}\left(\dot{y}_{1}-\dot{y}_{2}\right)}{2 \sqrt{y_{1}-y_{2}}}-S_{c} \ddot{y}_{2}\right) \\
& -a_{13} \sqrt{y_{1}-y_{2}} \\
& +a_{20} \sqrt{y_{2}-\left(\frac{a_{13} \sqrt{y_{1}-y_{2}}-S_{c} \dot{y}_{2}}{a_{32}}\right)^{2}} .
\end{aligned}\right.
$$

Fault detection and isolation. Additive actuator faults (bias) with different amplitudes are applied to both actuators and the consistency test (16) is used here. Equations (25) expressing the inputs in function of the flat outputs are required for detection. To control the conservatism induced by interval analysis due to the several occurrences of the variables $y_{1}, y_{2}, \dot{y}_{1}$ and $\dot{y}_{2}$ in the second equation of (25), we propose to use first (24) to estimate $x_{2}$. In addition, the derivative of $x_{2}$ is computed through the HOSM differentiator (14). Then, Eqn. (23b) is used to perform the inclusion test for $u_{2}$; thus only the first derivative of $y_{1}$ and $x_{2}$ is required to estimate $u_{1}$ and $u_{2}$.

As mentioned in Section 5, a robust differentiator built on the high order sliding modes technique provides the successive derivatives of the flat outputs, which yields

$$
\left\{\begin{array}{l}
\dot{y}_{1} \in\left[\dot{y}_{1_{\mathrm{est}}}-a c c_{\dot{y}_{1} \mathrm{est}}, \dot{y}_{1_{\mathrm{est}}}+a c c_{\dot{y}_{1} \mathrm{est}}\right] \\
\dot{x}_{2} \in\left[\dot{x}_{2_{\mathrm{est}}}-a c c_{\dot{x}_{2_{\mathrm{est}}}}, \dot{x}_{2_{\mathrm{est}}}+a c c_{\dot{x}_{2_{\mathrm{est}}}}\right],
\end{array}\right.
$$

where

$$
a c{\dot{y}_{1_{\mathrm{est}}}}=(0.002)^{\frac{1}{2}} \times\left(25.10^{-6}\right)^{\frac{1}{2}} \times 1=0.000224
$$

and

$$
a c c_{\dot{x}_{2_{\text {est }}}}=(0.002)^{1 / 2} \times(80 \mathrm{e}-8)^{1 / 2} \times 1=13 \mathrm{e}-6
$$

with a first order sliding-mode differentiator in both cases.

Table 1 sums up fault detection performances for the algorithm CSP Estimator applied to the three-tank system with the following faults:

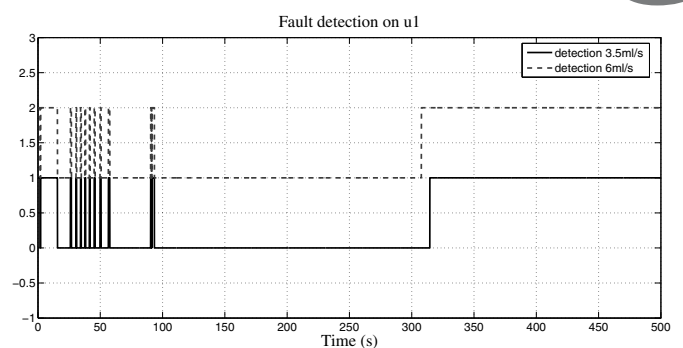

Fig. 3. Fault detection on $u_{1}$.

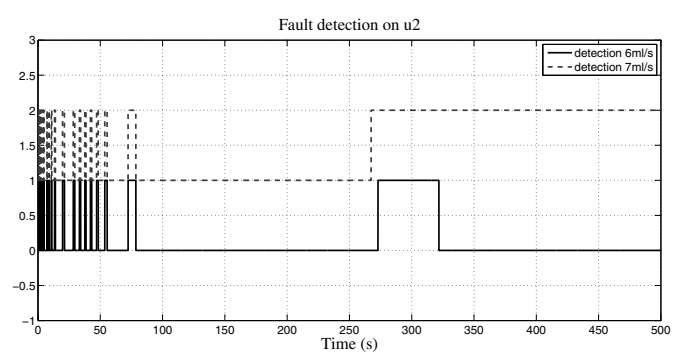

Fig. 4. Fault detection on $u_{2}$.

- additive bias on the first actuator $\left(u_{1}\right)$ appearing at $t=300 \mathrm{~s}$ with amplitudes $3.5 \mathrm{ml} / \mathrm{s}$ and $6 \mathrm{ml} / \mathrm{s}$;

- additive bias on the second actuator $\left(u_{2}\right)$ appearing at $t=250 \mathrm{~s}$ with amplitudes $6 \mathrm{ml} / \mathrm{s}$ and $7 \mathrm{ml} / \mathrm{s}$.

As we can see, the detection time decreases with respect to the fault amplitude growth. The detectability is stronger for the Actuator 1 and this could be explained by the fact that differential equation 23a leading it is relatively less complex than (23b) for Actuator 2 in the sense that for the variables $x_{1}, x_{2}$ and $x_{3}$ the occurrence number is lower than in 23b), which leads to less conservatism in the interval computations. Actually, in the case of a fault with an amplitude of $6 \mathrm{ml} / \mathrm{s}$ for the second actuator, the fault effects are not persistent. Note that the time interval $[0,80] \mathrm{s}$ corresponds to system transient behavior.

Fault identification. We can simultaneously estimate the admissible domain for the inputs $u_{1}$ and $u_{2}$ and the

Table 1. Fault detection performances.

\begin{tabular}{|l|l|l|l|l|}
\hline Actuator & Fault & $\begin{array}{l}\text { Amplitude } \\
(\%) \times u_{i \max }\end{array}$ & Amplitude & Delay \\
\hline \hline No. 1 & Bias & $8 \%$ & $3.5 \mathrm{ml} / \mathrm{s}$ & $14.5 \mathrm{~s}$ \\
& & $14 \%$ & $6 \mathrm{ml} / \mathrm{s}$ & $8 \mathrm{~s}$ \\
\hline No. 2 & Bias & $11 \%$ & $6 \mathrm{ml} / \mathrm{s}$ & $23 \mathrm{~s}$ \\
& & $13 \%$ & $7 \mathrm{ml} / \mathrm{s}$ & $17 \mathrm{~s}$ \\
\hline
\end{tabular}


fault (residual) estimated domain. Figures 5 and 6
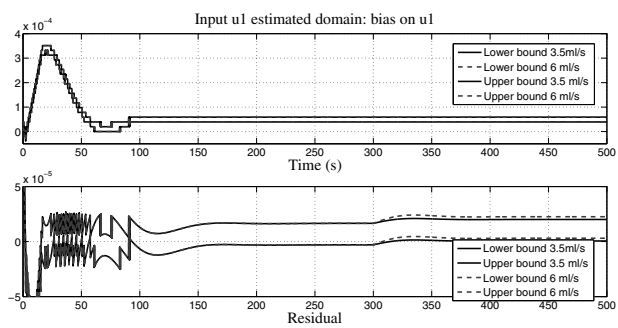

Fig. 5. Fault identification for $u_{1}$.

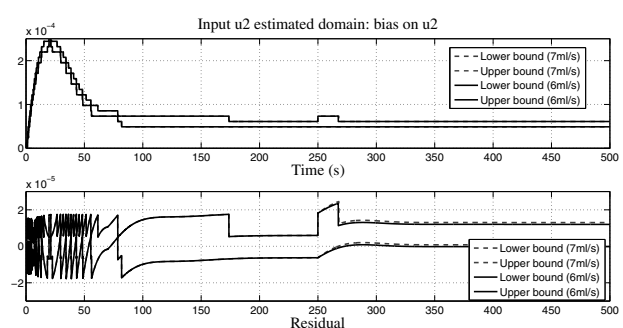

Fig. 6. Fault identification for $u_{2}$.

depict the kind of faults affecting the actuators. Moreover, the fault occurrence time is clearly shown $\left(t_{1}=300 \mathrm{~s}\right.$ for $u_{1}$ and $t_{2}=250 \mathrm{~s}$ for $u_{2}$ ) despite the existence of some detection delay time in both cases. The residual bounds, beyond the detection instant, give estimation for the domain of the fault amplitude. Depending on the differential equation leading to the actuator and the measurement derivative estimation, the pessimism induced around the fault amplitude may change.

\section{Minimum detectable fault.}

Actuator 1: The expression of the input is given by $u_{1}=S \dot{y}_{1}+a_{13} \sqrt{y_{1}-y_{2}}$. Given the bounds $a c c_{y_{1}}=$ $a c c_{y_{2}}=\bar{e}=0.002$ and $a c c_{\dot{y}_{1} \text { est }}=0.000224$, we have

$$
\begin{aligned}
f_{a_{1_{\text {min }}}} & =S \cdot\left|a c c_{\dot{y}_{1_{\text {est }}}}\right|+a_{13} \sqrt{a c c_{y_{1}}-a c c_{y_{2}}} \\
& =3.44 \cdot 10^{-6} \mathrm{mls}^{-1} .
\end{aligned}
$$

Actuator 2: The expression of $u_{2}$ is given by

$$
\begin{aligned}
u_{2}= & -\frac{\frac{2 S_{c}}{a_{32}}\left(a_{13} \sqrt{y_{1}-y_{2}}-S_{c} \dot{y}_{2}\right)}{a_{32}} \\
& \left(\frac{a_{13}\left(\dot{y}_{1}-\dot{y}_{2}\right)}{2 \sqrt{y_{1}-y_{2}}}-S_{c} \ddot{y}_{2}\right) \\
& -a_{13} \sqrt{y_{1}-y_{2}} \\
& +a_{20} \sqrt{y_{2}-\left(\frac{a_{13} \sqrt{y_{1}-y_{2}}-S_{c} \dot{y}_{2}}{a_{32}}\right)^{2}},
\end{aligned}
$$

which leads to

$$
\begin{aligned}
& f_{a_{\text {min }}} \\
& =-\frac{\frac{2 S_{c}}{a_{32}}\left(a_{13} \sqrt{a c c_{y_{1}}-a c c_{y_{2}}}-S_{c} a c c_{\dot{y}_{2_{\text {est }}}}\right.}{a_{32}} \\
& \cdot\left(\frac{a_{13}\left(a c c_{\dot{y}_{1} \text { est }}-a c c_{\dot{y}_{2} \text { est }}\right.}{2 \sqrt{a c c_{y_{1}}-a c c_{y_{2}}}}-S_{c} a c c_{\ddot{y}_{2}}\right) \\
& -a_{13} \sqrt{a c c_{y_{1}}-a c c_{y_{2}}} \\
& +a_{20} \sqrt{a c c_{y_{2}}-\frac{a_{13} \sqrt{a c c_{y_{1}}-a c c_{y_{2}}}-S_{c} a c c_{\dot{y}_{2_{\text {est }}}}}{a_{32}} .}
\end{aligned}
$$

Both flat outputs $y_{1}$ and $y_{2}$ are bounded by the value $0.002 \mathrm{~m}\left(a c c_{y_{1}}=a c c_{y_{2}}=2 \mathrm{~mm}\right)$. These uncertainties lead to a null denominator $\left(a c c_{y_{1}}-a c c_{y_{2}}=0\right)$ in the expression of $f_{a_{2_{\min }}}$. Since we consider that both outputs $y_{1}$ and $y_{2}$ are bounded by $a c c_{y_{1}}$ and $a c c_{y_{2}}$, we can extrapolate and consider $x_{2}$ as an output that would also be bounded by the same value $\left(a c c_{x_{2}}=2 \mathrm{~mm}\right)$, Then, from (23b), we have

$$
u_{2}=S * \dot{x}_{2}-a_{32} \sqrt{x_{3}-x_{2}}+a_{20} \sqrt{x_{2}},
$$

and the expression $f_{a_{2 m i n}}$ of the smallest detectable fault on the second actuator is given by

$$
\begin{aligned}
f_{a_{2_{\text {min }}}}= & S \cdot a c c_{\dot{x}_{2_{\mathrm{est}}}}-a_{32} \sqrt{a c c_{y_{2}}-a c c_{x_{2}}} \\
& +a_{20} \sqrt{a c c_{x_{2}}}=7.91 \mathrm{mls}^{-1} .
\end{aligned}
$$

Figure 4 shows that some faults with an amplitude lower than (28) can also be detected, which means that the expression given by (28) is conservative due to the estimation of the derivatives error bounds.

The drawback of the proposed technique is the computation time due to bisections in the set inversion procedure. It has been shown by Jaulin and Walter (1993) that the complexity of set inversion algorithms is exponential with respect to the unknown vector dimension $m$ (here, the dimension of the input vector):

$$
N=\left(\frac{w(U)}{\varepsilon}+1\right)^{m},
$$

where $U$ is the initial searching domain for the input $u$, $w(U)$ is the domain width and $\varepsilon$ is the bisection tolerance threshold. This technique can be implemented for systems with a low dimension for the input (or fault) vector.

\section{Concluding remarks}

In this paper, a new model-based fault diagnosis methodology with interval consistency techniques has been proposed for a class of nonlinear systems. The objective is to provide an original solution to actuator fault diagnosis for flat systems. The main important advantage 
of the technique is the possibility offered to generate a residual that gives the fault amplitude and satisfies robustness conditions under valid modeling hypotheses. Finally, the performance of the proposed strategy has been illustrated through a numerical example where no false alarm occurred during the detection phase. However, concerning an on-line implementation, the computational burden remains a drawback when dealing with high dimensional systems. An appealing direction for further investigations is the impact of parametric uncertainties and component faults that could lead to flatness property loss for a system. This is a topic of our current research.

\section{References}

Akhenak, A., Chadli, M., Maquin, D. and Ragot, J. (2003). Sliding mode multiple observer for fault detection and isolation, 42nd IEEE Conference on Decision and Control, Maui, HI, USA, Vol. 1, pp. 953-958.

Arangú, M. and Salido, M. (2011). A fine-grained arc-consistency algorithm for non-normalized constraint satisfaction problems, International Journal of Applied Mathematics and Computer Science 21(4): 733-744, DOI: 10.2478/v10006-011-0058-2.

Berdjag, D., Christophe, C., Cocquempot, V. and Jiang, B. (2006). Nonlinear model decomposition for robust fault detection and isolation using algebraic tools, International Journal of Innovative Computing, Information \& Control 2(6): 1337-1354.

Bernard, O. and Gouzé, J. (2004). Closed loop observers bundle for uncertain biotechnological models, Journal of Process Control 14(7): 765-774.

Besançon, G. and Zhang, Q. (2002). Further developments on nonlinear adaptive observers with application in fault detection, IFAC World Congress, Barcelona, Spain, Vol. 15, Part 1, pp. 19-22.

Bokor, J. and Balas, G. (2004). Detection filter design for LPV systems: A geometric approach, Automatica 40(3): 511-518.

Bokor, J. and Szab, Z. (2009). Fault detection and isolation in nonlinear systems, Annual Reviews in Control 33(2): 113-123.

Caccavale, F. and Villani, L. (2004). An adaptive observer for fault diagnosis in nonlinear discrete-time systems, American Control Conference, Boston, MA, USA, Vol. 3, pp. 2463-2468.

Chen, J. and Patton, R. (1999). Robust Model-Based Fault Diagnosis for Dynamic Systems, Kluwer Academic Publishers, Norwell, MA.

Chen, W., Saif, M. and Soh, Y. (2000). A variable structure adaptive observer approach for actuator fault detection and diagnosis in uncertain nonlinear systems, American Control Conference, Chicago, IL, USA, Vol. 4, pp. 2674-2678.

Corless, M. and Tu, J. (1998). State and input estimation for a class of uncertain systems, Automatica 34(6): 757-764.
De Persis, C. and Isidori, A. (2001). A geometric approach to nonlinear fault detection and isolation, IEEE Transactions on Automatic Control 46(6): 853-865.

De Persis, C. and Isidori, A. (2002). On the design of fault detection filters with game-theoretic-optimal sensitivity, International Journal of Robust and Nonlinear Control 12(8): 729-747.

Ding, S. (2008). Model-based Fault Diagnosis Techniques: Design Schemes, Algorithms, and Tools, Springer, Heidelberg/Berlin.

Ding, X. and Frank, P.M. (1993). An adaptive observer-based fault detection scheme for nonlinear dynamic systems, 12th IFAC World Congress, Sydney, Australia, Vol. 6, pp. 307-310.

Edwards, C. and Spurgeon, S.K. (2000). A sliding mode observer based FDI scheme for the ship benchmark, $E u$ ropean Journal of Control 6(4): 341-356.

Fagarasana, I., Ploix, S. and Gentil, S. (2004). Causal fault detection and isolation based on a set-membership approach, Automatica 40(12): 2099-2110.

Fliess, M., Levine, J., Martin, P. and Rouchon, P. (1992). Sur les systmes non linaires diffrentiellement plats, Comptes rendus de l'Acadmie des sciences, Srie 1: Mathmatiques 315(5): 619-624.

Gouzé, J., Rapaport, A. and Hadj-Sadok, M. (2000). Interval observers for uncertain biological systems, Ecological Modelling 133(1-2): 46-56.

Grenaille, S., Henry, D. and Zolghadri, A. (2008). A method for designing fault diagnosis filters for LPV polytopic systems, Journal of Control Science and Engineering (1): 1-11.

Ha, Q. and Trinh, H. (2004). State and input simultaneous estimation for a class of nonlinear systems, Automatica 40(10): 1779-1785.

Hakiki, K., Mazari, B., Liazid, A. and Djaber S. (2006). Fault reconstruction using sliding mode observers, American Journal of Applied Sciences 3(1): 1669-1674.

Hansen, R. (2004). Global Optimization Using Interval Analysis, 2nd Edn., CRC, New York, NY.

Henry, D. and Zolghadri, A. (2004). Robust fault diagnosis in uncertain linear parameter-varying systems, IEEE International Conference on Systems, Man and Cybernetics, The Hague, The Netherlands, Vol. 6, pp. 5165-5170.

Hermann, R. and Krener, A.J. (1977). Nonlinear controllability and observability, IEEE Transactions on Automatic Control 22(5): 728-740.

Hwang, I., Kim, S., Kim, Y. and Seah, C.E. (2010). A survey of fault detection, isolation, and reconfiguration methods, IEEE Transactions on Control Systems Technology 18(3): 636-653.

Ingimundarson, A., Bravo, J., Puig, V., Alamo, T. and Guerra, P. (2009). Robust fault detection using zonotope-based set-membership consistency test, International Journal of Adaptive Control and Signal Processing 23(4): 311-330.

Isermann, R. and Ball, P. (1997). Trends in the application of model-based fault detection and diagnosis of technical processes, Control Engineering Practice 5(5): 709-719. 
Jaulin, L. (2013). Combining interval analysis with flatness theory for state estimation of sailboat robots, Mathematics in Computer Sciences 6(4): 347-359.

Jaulin, L., Kieffer, M., Didrit, O. and Walter, E. (2001). Applied Interval Analysis, Springer, London.

Jaulin, L. and Walter, E. (1993). Set inversion via interval analysis for nonlinear bounded-error estimation, Automatica 29(4): 1053-1064.

Jiang, B., Staroswiecki, M. and Cocquempot, V. (2004). Fault estimation in nonlinear uncertain systems using robust/sliding-mode observers, IEE Proceedings Control Theory and Applications 151(1): 29-37.

Join, C., Sira-Ramirez, H. and Fliess, M. (2005). Control of an uncertain three-tank system via on-line parameter identification and fault detection, IFAC World Congress, Prague, Czech Republic.

Kabore, R. and Wang, H. (2001). Design of fault diagnosis filters and fault-tolerant control for a class of nonlinear systems, IEEE Transactions on Automatic Control 46(11): 1805-1810.

Korbicz, J., Kościelny, J.M., Kowalczuk, Z. and Cholewa, W. (Eds.) (2004). Fault Diagnosis: Models, Artificial Intelligence, Applications, Springer-Verlag, Berlin/Heidelberg.

Krantz, S. and Parks, H. (Eds.) (2002). The Implicit Function Theorem: History, Theory, and Applications, Birkhäuser, Boston, MA.

Lalami, A. and Combastel, C. (2006). Generation of set membership tests for fault diagnosis and evaluation of their worst case sensitivity, 6th IFAC Symposium on Fault Detection, Supervision and Safety of Technical Processes, Beijing, China, pp. 569-574.

Levant, A. (1998). Robust exact differentiation via sliding mode technique, Automatica 34(3): 379-384.

Levant, A. (2001). Higher order sliding modes and arbitrary order exact robust differentiation, European Control Conference, Porto, Portugal, pp. 996-1001.

Levant, A. (2003). Higher-order sliding modes, differentiation and output-feedback control, International Journal of Control 76(9): 924-941.

Liu, Y. (2009). Robust adaptive observer for nonlinear systems with unmodeled dynamics, Automatica 45(8): 1891-1895.

Moisan, M., Bernard, O. and Gouzé, J. (2009). Near optimal interval observers bundle for uncertain bioreactors, Automatica 45(1): 291-295.

Moisan, M. and Bernard, O. (2006). Robust interval observers for uncertain chaotic systems, 45th IEEE Conference on Decision and Control, San Diego, CA, USA, pp. 3712-3717.

Moore, R. (1966). Interval Analysis, Prentice-Hall, Englewood Cliffs, NJ

Neumaier, A. (2004). Complete search in continuous global optimization and constraint satisfaction, Acta Numerica 13: $271-369$.
Orani, N., Pisano, A. and Usai, E. (2009). Fault detection and reconstruction for a three-tank system via high-order sliding-mode observer, IEEE Multi-conference on Systems and Control (MSC), Saint-Petersburg, Russia pp. 1714-1719.

Poulsen, N. and Niemann, H. (2008). Active fault diagnosis based on stochastic tests, International Journal of Applied Mathematics and Computer Science 18(4): 487-496, DOI 10.2478/v10006-008-0043-6.

Puig, V. (2010). Fault diagnosis and fault tolerant control using set-membership approaches: Application to real case studies, International Journal of Applied Mathematics and Computer Science 20(4): 619-635, DOI: 10.2478/v10006-010-0046-y.

Puig, V., Quevedo, J., Escobet, T. and Stancu, R. (2003). Robust fault detection using linear interval observers, Proceedings of the 5th IFAC Symposium on Fault Detection, Supervision and Safety of Technical Processes, SAFEPROCESS 2003, Washington, DC, USA, pp. 609-614.

Ragot, J., Maquin, D. and Adrot, O. (2006). Parameter uncertainties characterisation for linear models, Computer Journal: 16-20.

Raïssi, T., Videau, G. and Zolghadri, A. (2010). Interval observer design for consistency checks of nonlinear continuous-time systems, Automatica 46(3): 518-527.

Sontag, E. and Wang, Y. (1991). I/O equations for nonlinear systems and observation spaces, 30th IEEE Conference on Decision and Control, Brighton, UK, pp. 720-725.

Stancu, A., Puig, V., Cuguer, P. and Quevedo, J. (2005) Benchmarking on approaches to interval observation applied to robust fault detection, in C. Jermann, A. Neumaier and D. Sam (Eds.), Global Optimization and Constraint Satisfaction, Lecture Notes in Computer Science, Vol. 3478, Springer, Berlin/Heidelberg, pp. 336-336.

Theilliol, D., Noura, H. and Ponsart, J. (2002). Fault diagnosis and accommodation of a three-tank system based on analytical redundancy, ISA Transactions 41(3): 365-382.

Waltz, D.L. (1975). Generating semantic descriptions from drawings of scenes with shadows, in P.H. Winston (Ed.), The Psychology of Computer Vision, McGraw-Hill, New York, NY, pp. 19-91.

Wang, H. (2003). Fault diagnosis and fault tolerant control for non-Gaussian stochastic systems with random parameters, in F. Caccavale and L. Villani (Eds.), Fault Diagnosis and Fault Tolerance for Mechatronic Systems: Recent Advances, Springer Tracts in Advanced Robotics, Vol. 1, Springer, Berlin/Heidelberg, pp. 59-84.

Wang, H. and Daley, S. (1996). Actuator fault diagnosis: An adaptive observer-based technique, IEEE Transactions on Automatic Control 41(7): 1073-1078.

Wang, H., Huang, Z. and Daley, S. (1997). On the use of adaptive updating rules for actuator and sensor fault diagnosis, $\mathrm{Au}$ tomatica 33(2): 217-225. 
Wang, H. and Lin, W. (2000). Applying observer based FDI techniques to detect faults in dynamic and bounded stochastic distributions, International Journal of Control 73(15): 1424-1436.

Wang, H. and Noriega, R. (2001). Fault diagnosis for unknown non-linear systems via neural networks and its comparisons and combinations with recursive least-squares based techniques, Proceedings of the Institution of Mechanical Engineers, Part I: Journal of Systems and Control Engineering 215(3): 261-278.

Yan, X. and Edwards, C. (2008). Adaptive sliding-mode-observer-based fault reconstruction for nonlinear systems with parametric uncertainties, IEEE Transactions on Industrial Electronics 55(11): 4029-4036.

Zhang, Q., Basseville, M. and Benveniste, A. (1998). Fault detection and isolation in nonlinear dynamic systems: A combined inputoutput and local approach, Automatica 34(11): 1359-1373.

Zolghadri, A., Henry, D. and Monsion, M. (1996). Design of nonlinear observers for fault diagnosis: A case study, Control Engineering Practice 4(11): 1535-1544.

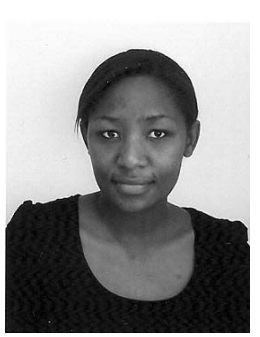

Ramatou Seydou obtained her Master degree at the Aeronautical Maintenance Institute, Bordeaux University, France, in 2009. She is currently a Ph.D. student at the IMS Lab, Bordeaux University. The topic of her thesis is the design and development of set membership techniques and their application to fault detection and diagnosis.

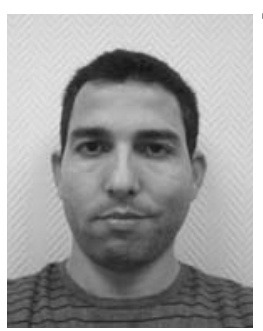

Tarek Rassi received the Engineer degree from Tunis National Engineering School in 2000, the DEA in automatic control from Lille Centrale Graduate School in 2001 and the Ph.D. degree from University Paris XII in 2004. From 2005 to 2011 he was an associate professor at the University of Bordeaux. He is currently at the $\mathrm{Na}$ tional Conservatory of Arts and Crafts, Paris, France. He is a member of the IFAC TC on Modelling, Identification and Signal Processing. His research interests include fault detection and isolation, nonlinear systems estimation, interval analysis.

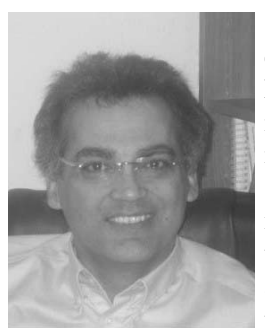

Ali Zolghadri is a full professor of control engineering with the University of Bordeaux, France. His main research interest is narrowing the gap between real world control engineering requirements and theoretical analysis and design techniques. His areas of expertise include model-based fault diagnosis, fault-tolerant control and guidance, health management and operational autonomy for complex safety-critical systems. He has published around 150 contributions including journal articles, book chapters and communications. He is a co-holder of 5 patents.

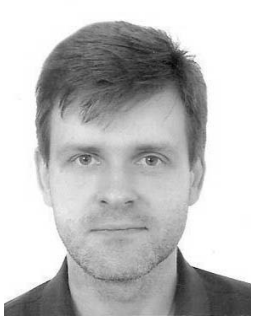

Denis Efimov received the Ph.D. degree in automatic control from the Saint-Petersburg State Electrical Engineering University (Russia) in 2001, and the Dr.Sc. degree in automatic control in 2006 from the Institute for Problems of Mechanical Engineering of the Russian Academy of Sciences (Saint-Petersburg). From 2000 to 2009 he was a research assistant at the Institute for Problems of Mechanical Engineering, RAS, Control of Complex Systems Laboratory. From 2006 to 2011 he was working in the LSS (Supélec, France), the Monteore Institute (University of Liege, Belgium) and the Automatic Control Group at the IMS Lab (University of Bordeaux I, France). In 2011 he joined the Non-A team at the INRIA Lille Center. He is a member of the IFAC TC on Adaptive and Learning Systems and a senior member of the IEEE. His main research interests include nonlinear oscillation analysis, observation and control and switched and hybrid system stability.

\section{Appendix}

Table A1. Three-tank system parameters.

\begin{tabular}{|c|c|c|c|}
\hline Parameter & Description & Units & $\begin{array}{l}\text { Nominal } \\
\text { values }\end{array}$ \\
\hline$S$ & $\begin{array}{ll}\text { Tank surface } \\
\text { area }\end{array}$ & $\overline{m^{2}}$ & 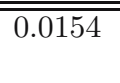 \\
\hline $\boldsymbol{S}_{n}$ & $\begin{array}{ll}\text { Pipe } & \text { surface } \\
\text { area } & \\
\end{array}$ & $\mathrm{m}^{2}$ & $5 \cdot 10^{-5}$ \\
\hline$a_{13}$ & $a_{z 13} S_{n} \sqrt{2 g}$ & - & \\
\hline$a_{20}$ & $a_{z 20} S_{n} \sqrt{2 g}$ & - & \\
\hline$a_{32}$ & $a_{z 32} S_{n} \sqrt{2 g}$ & - & \\
\hline $\boldsymbol{a}_{z 13}$ & & - & 0.6 \\
\hline $\boldsymbol{a}_{z 20}$ & & - & 0.8 \\
\hline $\boldsymbol{a}_{z 32}$ & & - & 0.6 \\
\hline$g$ & $\begin{array}{l}\text { Gravity } \\
\text { constant }\end{array}$ & $\mathrm{N} / \mathrm{m}^{2}$ & 9.81 \\
\hline $\begin{array}{l}\boldsymbol{h}_{1 \max } \\
\boldsymbol{h}_{2 \max } \\
\boldsymbol{h}_{3 \max }\end{array}$ & $\begin{array}{l}\text { Maximum } \\
\text { levels in tank } \\
1,2 \text { and } 3\end{array}$ & $\mathrm{~m}$ & $\begin{array}{l}0.5 \\
0.3 \\
0.4\end{array}$ \\
\hline $\begin{array}{l}\boldsymbol{Q}_{1 \max } \\
\boldsymbol{Q}_{2 \max }\end{array}$ & $\begin{array}{lr}\begin{array}{l}\text { Input } \\
\text { rates }\end{array} & \text { (steady } \\
\text { state) } & \end{array}$ & $\mathrm{mls}^{-1}$ & $\begin{array}{l}42 \\
55\end{array}$ \\
\hline
\end{tabular}

Received: 24 June 2011 Revised: 28 November 2011 Re-revised: 17 April 2012 